\title{
Finite-time stabilization of switched nonlinear singular systems with asynchronous switching
}

\author{
Jing Wang ${ }^{1 *}$ (D) and Xingtao Wang
}

\section{"Correspondence:}

16b912020@stu.hit.edu.cn 'Department of Mathematics, Harbin Institute of Technology, Xidazhi Road, Harbin, 150000, P.R. China

\section{Springer}

\begin{abstract}
This paper is concerned with the finite-time stabilization of a class of switched nonlinear singular systems under asynchronous control. Asynchronism here refers to the delays in switching between the controller and the subsystem. First, the dynamic decomposition technique is used to prove that such a switched singular system is regular and impulse-free. Secondly, based on the state solutions of the closed-loop system in the matched time period and the mismatched time period of the system instead of constructing a Lyapunov function, the sufficient conditions for the finite-time stability of the asynchronous switched singular system are given, there is no limit to the stability of subsystems. Then, the mode-dependent state feedback controller that makes the original system stable is derived in the form of strict linear matrix inequalities. Finally, numerical examples are given to verify the feasibility and validity of the results.
\end{abstract}

Keywords: Switched singular systems; Finite-time stabilization; Asynchronous switching; Average dwell time; Linear matrix inequality

\section{Introduction}

A switched system is a class of hybrid system consisting of several continuous or discrete dynamic subsystems and a given switching rule. When simulating complex models, switched systems often have an advantage over a single system, so they are widely used in many fields such as switching power converters, aircraft and air-traffic control, see [15]. In recent years, many studies on switched systems have emerged, see [6] and [7, 8] and references therein. Most studies on switched systems are concerned with Lyapunov global asymptotic stability. However, in practice, we need the system to be stable within a finitetime interval instead of an infinite interval. The finite-time stability problem of a switched system has been discussed in [9-12]. Therefore, it is more valuable to study the transient performance of the system in a finite-time interval than Lyapunov asymptotic stability in some situations. The difference between the concept of finite-time stability and Lyapunov stability is mainly manifested in two aspects: one is that finite-time stability analyzes the system within a limited time interval; the other is that finite-time stability requires preset boundaries of system variables.

(c) The Author(s) 2021. This article is licensed under a Creative Commons Attribution 4.0 International License, which permits use sharing, adaptation, distribution and reproduction in any medium or format, as long as you give appropriate credit to the original author(s) and the source, provide a link to the Creative Commons licence, and indicate if changes were made. The images or other third party material in this article are included in the article's Creative Commons licence, unless indicated otherwise in a credit line to the material. If material is not included in the article's Creative Commons licence and your intended use is not permitted by statutory regulation or exceeds the permitted use, you will need to obtain permission directly from the copyright holder. To view a copy of this licence, visit http://creativecommons.org/licenses/by/4.0/. 
A switched singular system means that the system contains at least one singular subsystem. These systems widely exist in power systems, networked control systems, robotics and other practical systems [13-15]. Therefore, the study of switched singular systems has attracted the attention of many workers, and has achieved rich research results [1618]. Compared with general switched systems, the stability analysis and controller design of switched singular systems are more complicated due to the problems of regularity, uniform initial state and impulse-mode cancelation. When more detailed and precise models are pursued, models of nonlinear rather than linear singular systems are established. It is inevitable that switching signals will take a certain amount of time in the transmission process, as even modern technology can not completely eliminate the time delay. Like the butterfly effect, even a small delay of the controller may have a great influence on the system. Thus, in order to simulate a more realistic real system, many workers focus their research on meaningful asynchronous controllers [19-22].

In the previous paper on switched singular systems [23], based on the equivalent dynamics-decomposition form, the exact description of the state jump is characterized at the moment of system switching. On the one hand, this state jump comes from the switching law of piecewise-constant values, and on the other hand, it comes from the constraint of algebraic equations. On the basis of the refined description for state jumps proposed above, the finite-time stabilization problem of switched linear singular systems has been considered in [24] without considering the occurrence of asynchronism. Some conditions to ensure that the state remains in a bounded region have been derived via the Lyapunov approach. The finite-time stability problem and finite-time bounded problem of switched singular systems with unstable subsystems have been presented by the authors in [25]. With the help of illustrative examples, the criterion given in [25] provides less conservative results than the approach given in [24]. For the vast majority of methods used to solve the finite-time stability of switched systems, Lyapunov methods have been proven to be one of the most efficient approaches [5, 26, 27]. Moreover, the Lyapunov function method is also a very effective tool when studying fractional-order systems, see [28-31]. The efficiency of those methods, however, depends crucially on appropriate construction of the Lyapunov-Krasovskii (L-K) functions. Since there is no uniform method to construct $\mathrm{L}-\mathrm{K}$ functions, it is not easy to construct suitable $\mathrm{L}-\mathrm{K}$ functions for different systems. Hence, we are curious about one thing: can we solve the problem of finite-time stability of switched singular systems under asynchronous control without using the Lyapunov function method? This is the first motivation of this research.

In fact, the solution of the state equation of the system is an intuitive and useful tool in studying the stability of the system, yet few workers use it. There are two main reasons for this phenomenon. On the one hand, the structure and state equation of the switching system are complex, the switching signals are constantly changing. Meanwhile, the subsystems are alternating so it is difficult to obtain the state solution of the system. On the other hand, even if the state solution is obtained, it is difficult to find effective analysis tools and methods. Thus, starting from the original solution of the system and combining the model with the mode-dependent average dwell time to study the asynchronous problem of a switched singular system has not been given enough attention, which is the second motivation of this paper.

The objective of this paper is twofold. The first is to find the appropriate switching law to make the system stable in finite time. The other is to find the specific form of an asyn- 
chronous controller that can be solved. Based on the problems raised above, the contributions of this paper are as follows.

(i) The regular and impulse-free properties of switched singular systems is proved based on the dynamic decomposition technique and there is no requirement that all subsystems must be stable. Then, the finite-time stability (FTS) problem of a switched singular system is transformed into the FTS problem of reduced-order switched systems.

(ii) In contrast to [24, 25, 32], we do not construct any Lyapunov functions in our research. Starting with the state-equation solution of the switched system with nonlinear disturbance and taking the switching time point as the boundary, the operation time period of each switched system is analyzed, and the state solutions of the closed-loop system in the matched time period and the mismatched time period are given, and the state solutions of the whole time period are obtained by alternating iterative derivation.

(iii) Based on the mathematical derivation and analysis of the state solution, and combined with the average dwell time method, the sufficient conditions for the FTS of the closed-loop switched singular system are obtained. Then, sufficient conditions for the system to be FTS are given in the form of strict linear matrix inequality and the gain matrix form of the controller is presented. Compared with [25], sufficient conditions with less conservatism can be obtained to determine the FTS of a switched singular system.

The rest of this paper is organized as follows. In Sect. 2, definitions and lemmas useful for the proof of theorems in this paper are listed. Section 3 presents the main results. Based on the decomposition transformation of the original system and taking the asynchronous controller into account, sufficient conditions for finite-time stability of switched singular systems are given. The proof process is concise and to the point. Two specific examples along with numerical and simulation results are provided in Sect. 4 . Section 5 gives the conclusion of the work of this paper.

Notations: The notations used in this paper are fairly standard. $\mathbb{R}^{n}$ denotes the $n$ dimensional Euclidean space over the reals, $\mathbb{R}^{m \times n}$ is the set of all $m \times n$ real matrices. $N^{+}$represents all positive integer sets. " $*$ " stands for the symmetric term in a symmetric matrix. $\operatorname{Re}(A)$ represents the real parts of the eigenvalues of matrix $A . P>0(P<0)$ means that $P$ is real symmetric and positive-definite (negative-definite). Matrix $P>Q(P \geq Q)$ is equivalent to $P-Q>0(P-Q \geq 0) . \lambda_{\max }(P)\left(\lambda_{\min }(P)\right)$ denotes the maximum (minimum) eigenvalue of $P$, and $\|\cdot\|$ is the Euclidean norm.

\section{Problem statement and preliminaries}

Consider a class of nonlinear switched singular systems described by the following equation:

$$
\left\{\begin{array}{l}
E_{\sigma(t)} \dot{x}(t)=A_{\sigma(t)} x(t)+B_{\sigma(t)} u(t)+f_{\sigma(t)}(t, x(t)), \\
x\left(t_{0}\right)=x_{0} .
\end{array}\right.
$$

where $x(t) \in \mathbb{R}^{n}, u(t) \in \mathbb{R}^{m}$ are the state vector and control input, the index $\sigma(t):[0, \infty) \rightarrow$ $\mathcal{N}=\{1,2, \ldots, \mathbf{N}\}$ is a piecewise right-continuous function of time $t$ or $x(t)$, where $\mathbf{N} \in N^{+}$ is the number of subsystems. The switching sequence satisfies $t_{0}<t_{1}<t_{2}<\cdots$, when $t \in\left[t_{i}, t_{i+1}\right)$ and $\sigma(t)=l_{i} \in \mathcal{N}$, we say that subsystem $l_{i}$ is activated. For all $\sigma(t)=l_{i} \in \mathcal{N}$, $E_{\sigma(t)}, A_{\sigma(t)}, B_{\sigma(t)}$ are known constant matrices with appropriate dimensions. Meanwhile, 
$E_{\sigma(t)}$ is a singular matrix and satisfying $\operatorname{rank} E_{\sigma(t)}=r<n \cdot f_{\sigma(t)}(t, x(t))$ is a continuously differentiable nonlinear perturbation function on $x(t)$, and $f_{\sigma(t)}(t, 0)=0$ and satisfies the following quadratic constraint

$$
f_{\sigma(t)}^{\mathrm{T}}(t, x(t)) f_{\sigma(t)}(t, x(t)) \leq \omega^{2} x^{\mathrm{T}}(t) W_{\sigma(t)}^{\mathrm{T}} W_{\sigma(t)} x(t) .
$$

In practical engineering applications, because the sensor identify subsystem and the corresponding controller will take some time, there will be a switching time delay in the controller, which results in switching asynchrony between them. Therefore, in this paper, the following form of controller is considered

$$
u(t)=K_{\sigma(t-\tau(t))} x(t)
$$

where $\tau(t)$ is the switching delay of the controller relative to the subsystem while meeting $0<\tau\left(t_{i}\right) \leq \bar{\tau} \leq t_{i+1}-t_{i}$. Here, without loss of generality [33, 34], the upper bound of the switching delay is known in advance. By substituting this expression into formula (1), we get the following closed-loop system expression

$$
\left\{\begin{array}{l}
E_{\sigma(t)} \dot{x}(t)=\left(A_{\sigma(t)}+B_{\sigma(t)} K_{\sigma(t-\tau(t))}\right) x(t)+f_{\sigma(t)}(t, x(t)), \\
x\left(t_{0}\right)=x_{0} .
\end{array}\right.
$$

The purpose here is to design a state feedback controller (3) such that the loop-closed system (4) is admissible. The switching time series of the controller is $t_{0}<t_{1}+\tau\left(t_{1}\right)<\cdots<$ $t_{i}+\tau\left(t_{i}\right)<\cdots$. Meanwhile, $\tilde{t}_{i}$ is defined as $t_{i}+\tau\left(t_{i}\right)$. Further, system (4) can be written in the following form

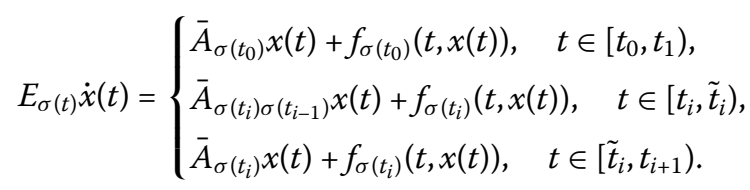

For simplicity, we use the subscripts $l_{i}$ and $l_{i-1}$ to substitute for $\sigma\left(t_{i}\right)$ and $\sigma\left(t_{i-1}\right)$. We use $\tilde{\sigma}\left(t_{i}\right)=\sigma\left(t_{i}\right) \sigma\left(t_{i-1}\right)=l_{i} l_{i-1}, t \in\left[t_{i}, \tilde{t}_{i}\right), \tilde{\sigma}\left(t_{i}\right)=\sigma\left(t_{i}\right)=l_{i}, t \in\left[\tilde{t}_{i}, t_{i+1}\right)$, thus $\bar{A}_{l_{i} l_{i-1}}=A_{l_{i}}+B_{l_{i}} K_{l_{i-1}}$, $\bar{A}_{l_{i}}=A_{l_{i}}+B_{l_{i}} K_{l_{i}}$. The above formula can be abbreviated as

$$
E_{\tilde{\sigma}(t) \dot{x}}(t)=\bar{A}_{\tilde{\sigma}(t)} x(t)+f_{\sigma(t)}(t, x(t)) .
$$

In order to prove the theorem, we need some definitions and lemmas.

Definition 2.1 ([25]) For the switching signal $\sigma(t)$ of system (1) and any $t_{2}>t_{1} \geq 0$, let $N_{\sigma l_{i}}\left(t_{1}, t_{2}\right)$ denote the switched numbers of the $l_{i} t h$ subsystem over $\left(t_{1}, t_{2}\right), T_{l_{i}}\left(t_{1}, t_{2}\right)$ as the sum of the running time of the $l_{i}$ th mode, if

$$
N_{\sigma l_{i}}\left(t_{1}, t_{2}\right) \leq N_{0 l_{i}}+\frac{T_{l_{i}}\left(t_{1}, t_{2}\right)}{\tau_{a l_{i}}},
$$

holds for $\tau_{a l_{i}}>0, N_{0 l_{i}} \geq 0$, then $\tau_{a l_{i}}$ is called the mode-dependent average dwell time and $N_{0 l_{i}}$ is called a chatter bound of the switching signal $\sigma(t)$. 
Definition 2.2 ([35]) For every $l_{i} \in \mathcal{N}$, the pair $\left(E_{l_{i}}, A_{l_{i}}\right)$ in (1) is said to be

(1) regular if $\operatorname{det}\left(s E_{l_{i}}-A_{l_{i}}\right)$ is not identically zero;

(2) impulse-free if $\operatorname{deg}\left(\operatorname{det}\left(s E_{l_{i}}-A_{l_{i}}\right)\right)=\operatorname{rank}\left(E_{l_{i}}\right)$.

Definition 2.3 ([24]) For given three positive numbers $c_{1}, c_{2}, T$, with $c_{1}<c_{2}$, a positivedefinite matrix $R>0$ and a given switching signal $\sigma(t) \in \mathcal{N}$, the switched nonlinear singular system (1) is said to be finite-time stabilized under an appropriate control input $u(t)$ with respect to $\left(c_{1}, c_{2}, T, R, \sigma\right)$, if

$$
x^{\mathrm{T}}(0) E_{\sigma\left(t_{0}\right)}^{\mathrm{T}} R E_{\sigma\left(t_{0}\right)} x(0) \leq c_{1} \quad \Rightarrow \quad x^{\mathrm{T}}(t) E_{\sigma(t)}^{\mathrm{T}} R E_{\sigma(t)} x(t) \leq c_{2}, \quad \forall t \in[0, T] .
$$

Lemma 2.1 Let $\mathcal{M}=\left[\begin{array}{ll}\mathcal{A} & \mathcal{B} \\ \mathcal{C} & \mathcal{D}\end{array}\right]$, where $\mathcal{A}, \mathcal{B}, \mathcal{C}$ and $\mathcal{D}$ are any real given matrices with appropriate dimensions such that $\mathcal{M}+\mathcal{M}^{\mathrm{T}}<0$. Then, $\mathcal{D}$ is nonsingular and $\mathcal{A}+\mathcal{A}^{\mathrm{T}}-\mathcal{B D}^{-1} \mathcal{C}-$ $\mathcal{C}^{\mathrm{T}} \mathcal{D}^{-\mathrm{T}} \mathcal{B}^{\mathrm{T}}<0$.

Lemma 2.2 ([36]) Let $u, v$ and $w$ be nonnegative piecewise-continuous functions on $[0,+\infty)$ for which the inequality

$$
u(t) \leq c+\int_{a}^{t}(u(s) v(s)+w(s)) \mathrm{d} s, \quad \forall t \geq a,
$$

holds, where $a$ and $c$ are nonnegative constants. Then,

$$
u(t) \leq c \mathrm{e}^{\int_{a}^{t} v(s) \mathrm{d} s}+r \mathrm{e}^{\int_{a}^{t}\left(\nu(s)+\frac{w(s)}{r(s)}\right) \mathrm{d} s}, \quad \forall t \geq a, \forall r>0 .
$$

\section{Main results}

In this section, the decomposition technique and average dwell-time method are combined together to investigate the finite-time stabilization problems for the closed-loop system (6). Since $\operatorname{rank} E_{l_{i}}=r<n$, there exist two invertible matrices $M_{l_{i}}$ and $N_{l_{i}}$ such that

$$
\begin{aligned}
& x(t)=N_{l_{i}}\left[\begin{array}{l}
\bar{x}_{1}(t) \\
\bar{x}_{2}(t)
\end{array}\right], \quad M_{l_{i}} E_{l_{i}} N_{l_{i}}=\left[\begin{array}{cc}
I_{r} & 0 \\
0 & 0
\end{array}\right], \quad M_{l_{i}} \bar{A}_{l_{i}} N_{l_{i}}=\left[\begin{array}{ll}
A_{l_{i} 11} & A_{l_{i} 12} \\
A_{l_{i} 1} & A_{l_{2} 22}
\end{array}\right], \\
& M_{l_{i} f_{l_{i}}}=\left[\begin{array}{l}
f_{l_{i} 1} \\
f_{l_{i} 2}
\end{array}\right],
\end{aligned}
$$

where $\bar{x}_{1}(t) \in \mathbb{R}^{r}, \bar{x}_{2}(t) \in \mathbb{R}^{n-r}$. Then, equation (6) can be converted into

$$
\left\{\begin{array}{l}
\dot{\bar{x}}_{1}(t)=A_{\tilde{\sigma} 11} \bar{x}_{1}(t)+A_{\tilde{\sigma} 12} \bar{x}_{2}(t)+f_{\sigma 1}(t, x(t)), \\
0=A_{\tilde{\sigma} 21} \bar{x}_{1}(t)+A_{\tilde{\sigma} 22} \bar{x}_{2}(t)+f_{\sigma 2}(t, x(t)) .
\end{array}\right.
$$

Suppose $A_{\tilde{\sigma} 22}$ is nonsingular, the following formula can be further obtained

$$
\left\{\begin{array}{l}
\dot{\bar{x}}_{1}(t)=A_{\tilde{\sigma} 11} \bar{x}_{1}(t)+A_{\tilde{\sigma} 12} \bar{x}_{2}(t)+f_{\sigma 1}(t, x(t)), \\
\bar{x}_{2}(t)=-A_{\tilde{\sigma} 22}^{-1} A_{\tilde{\sigma} 21} \bar{x}_{1}(t)-A_{\tilde{\sigma} 22}^{-1} f_{\sigma 2}(t, x(t)) .
\end{array}\right.
$$


Thus, system (6) can be rewritten as

$$
\left\{\begin{array}{l}
\dot{\bar{x}}_{1}(t)=\bar{A}_{\tilde{\sigma} 1} \bar{x}_{1}(t)+h_{\sigma 1}(t, x(t)), \\
\bar{x}_{2}(t)=\bar{A}_{\tilde{\sigma} 2} \bar{x}_{1}(t)+h_{\sigma 2}(t, x(t)),
\end{array}\right.
$$

where $\bar{A}_{\tilde{\sigma} 1}=A_{\tilde{\sigma} 11}-A_{\tilde{\sigma} 12} A_{\tilde{\sigma} 22}^{-1} A_{\tilde{\sigma} 21}, \bar{A}_{\tilde{\sigma} 2}=-A_{\tilde{\sigma} 22}^{-1} A_{\tilde{\sigma} 21}, h_{\sigma 1}=-A_{\tilde{\sigma} 12} A_{\tilde{\sigma} 22}^{-1} f_{\sigma 2}(t, x(t))+$ $f_{\sigma 1}(t, x(t)), h_{\sigma 2}=-A_{\tilde{\sigma} 22}^{-1} f_{\sigma 2}(t, x(t))$. At the same time, suppose there is a constant $\delta>0$ such that $h_{\sigma(t) 1}(t, x(t))$ satisfies the following inequality

$$
\left\|h_{\sigma(t) 1}(t, x(t))\right\| \leq \delta\left\|\bar{x}_{1}(t)\right\|, \quad \forall \sigma(t) \in \mathcal{N}
$$

This article follows the previous definition, where $\bar{x}_{1}(t)$ is called the slow system variable and $\bar{x}_{2}(t)$ is called the fast subsystem variable.

Remark 3.1 It should be noted that the dynamics-decomposition form is not unique because the choice of matrices $M_{l_{i}}, N_{l_{i}}$ is not unique. According to the proof of Theorem 3.1 in reference [37], it can be seen that the properties of the system solution remain unchanged after the coefficient matrix of the system is transformed. Therefore, the regular and impulse-free nature of the solutions of (1) and (11) can be derived from each other. Some similar definitions about the pair $\left(E_{l_{i}}, A_{l_{i}}\right)$ appear in Theorem 1 in [35] and Definition 1 in [38].

Remark 3.2 As stated in [39], finite-time stability and Lyapunov stability are two independent concepts. The former describes the local properties of the system state, and the latter describes the global asymptotic behavior of the system solution. These two properties cannot be deduced from each other. The upper bound $T$ of the system running time is determined according to the specific situation in a practical application. Therefore, in this study, $T$ is a known value given in advance. At the same time, the average dwell time should be as small as possible to reduce conservatism.

The proof will be divided into two steps. Let us start with the observation that system (6) is regular and impulse free.

Theorem 3.1 Consider the switched singular system (6), given constants $\alpha_{l_{i}}>0$, if there exist nonsingular matrices $P_{l_{i}}, \forall l_{i} \in \mathcal{N}$ such that

$$
\begin{aligned}
& E_{l_{i}}^{\mathrm{T}} P_{l_{i}}=P_{l_{i}}^{\mathrm{T}} E_{l_{i}} \geq 0, \\
& {\left[\begin{array}{ccc}
\Phi_{11 l_{i}} & P_{l_{i}}^{\mathrm{T}} & W_{l_{i}}^{\mathrm{T}} \\
* & -I & 0 \\
* & * & -\gamma I
\end{array}\right]<0,}
\end{aligned}
$$

where $\Phi_{11 l_{i}}=\bar{A}_{l_{i}}^{\mathrm{T}} P_{l_{i}}+P_{l_{i}}^{\mathrm{T}} \bar{A}_{l_{i}}-2 \alpha_{l_{i}} E_{l_{i}}^{\mathrm{T}} P_{l_{i}}, \gamma=\omega^{-2}$ hold, then the pair $\left(E_{l_{i}}, A_{l_{i}}\right)$ in system (6) is regular and impulse free and system (6) has a unique solution in the neighborhood of an equilibrium point. 
Proof From condition (13), we have

$$
N_{l_{i}}^{\mathrm{T}} E_{l_{i}}^{\mathrm{T}} M_{l_{i}}^{\mathrm{T}}\left(M_{l_{i}}^{\mathrm{T}}\right)^{-1} P_{l_{i}} N_{l_{i}}=N_{l_{i}}^{\mathrm{T}} P_{l_{i}}^{\mathrm{T}} M_{l_{i}}^{-1} M_{l_{i}} E_{l_{i}} N_{l_{i}} \geq 0
$$

Taking $\bar{P}_{l_{i}}=\left(M_{l_{i}}^{\mathrm{T}}\right)^{-1} P_{l_{i}} N_{l_{i}}=\left[\begin{array}{ll}P_{l_{i} 11} & P_{l_{i} 12} \\ P_{l_{i} 21} & P_{l_{i} 22}\end{array}\right]$. Thus, from $\left[\begin{array}{cc}I_{r} & 0 \\ 0 & 0\end{array}\right] \bar{P}_{l_{i}}=\bar{P}_{l_{i}}^{\mathrm{T}}\left[\begin{array}{cc}I_{r} & 0 \\ 0 & 0\end{array}\right]$, we can obtain $P_{l_{i} 11}>$ $0, P_{l_{i} 12}=0$. We can conclude from (14) that

$$
N_{l_{i}}^{\mathrm{T}} \bar{A}_{l_{i}}^{\mathrm{T}} M_{l_{i}}^{\mathrm{T}}\left(M_{l_{i}}^{\mathrm{T}}\right)^{-1} P_{l_{i}} N_{l_{i}}+N_{l_{i}}^{\mathrm{T}} P_{l_{i}}^{\mathrm{T}} M_{l_{i}}^{-1} M_{l_{i}} \bar{A}_{l_{i}} N_{l_{i}}-2 \alpha_{l_{i}} N_{l_{i}}^{\mathrm{T}} E_{l_{i}}^{\mathrm{T}} M_{l_{i}}^{\mathrm{T}}\left(M_{l_{i}}^{\mathrm{T}}\right)^{-1} P_{l_{i}} N_{l_{i}}<0 .
$$

Substituting (9) into the above formula, we have

$$
\begin{gathered}
{\left[\begin{array}{cc}
A_{l_{i} 11} & A_{l_{i} 12} \\
A_{l_{i} 21} & A_{l_{i} 22}
\end{array}\right]^{\mathrm{T}}\left[\begin{array}{cc}
P_{l_{i} 11} & 0 \\
P_{l_{i} 21} & P_{l_{i} 22}
\end{array}\right]+\left[\begin{array}{cc}
P_{l_{i} 11} & 0 \\
P_{l_{i} 21} & P_{l_{i} 22}
\end{array}\right]^{\mathrm{T}}\left[\begin{array}{cc}
A_{l_{i} 11} & A_{l_{i} 12} \\
A_{l_{i} 21} & A_{l_{i} 22}
\end{array}\right]} \\
-2 \alpha_{l_{i}}\left[\begin{array}{cc}
I_{r} & 0 \\
0 & 0
\end{array}\right]\left[\begin{array}{cc}
P_{l_{i} 11} & 0 \\
P_{l_{i} 21} & P_{l_{i} 22}
\end{array}\right]<0,
\end{gathered}
$$

and finally that

$$
\left[\begin{array}{cc}
\operatorname{Sym}\left(A_{l_{i} 11}^{\mathrm{T}} P_{l_{i} 11}\right)+\operatorname{Sym}\left(A_{l_{i} 21}^{\mathrm{T}} P_{l_{i} 21}\right)-2 \alpha_{l_{i}} P_{l_{i} 11} & A_{l_{i} 21}^{\mathrm{T}} P_{l_{i} 22}+P_{l_{i} 21}^{\mathrm{T}} A_{l_{i} 22}+P_{l_{i} 11}^{\mathrm{T}} A_{l_{i} 12} \\
A_{l_{i} 22}^{\mathrm{T}} P_{l_{i} 21}+P_{l_{i} 22}^{\mathrm{T}} A_{l_{i} 21}+A_{l_{i} 12}^{\mathrm{T}} P_{l_{i} 11} & A_{l_{i} 22}^{\mathrm{T}} P_{l_{i} 22}+P_{l_{i} 22}^{\mathrm{T}} A_{l_{i} 22}
\end{array}\right]<0 .
$$

Let

$$
\mathcal{M}=\left[\begin{array}{cc}
P_{l_{i} 11}^{\mathrm{T}} A_{l_{i} 11}+P_{l_{i} 21}^{\mathrm{T}} A_{l_{i} 21}-\alpha_{l_{i}} P_{l_{i} 11} & A_{l_{i} 21}^{\mathrm{T}} P_{l_{i} 22} \\
A_{l_{i} 22}^{\mathrm{T}} P_{l_{i} 21}+A_{l_{i} 12}^{\mathrm{T}} P_{l_{i} 11} & A_{l_{i} 22}^{\mathrm{T}} P_{l_{i} 22}
\end{array}\right]
$$

according to Lemma 2.1, it follows that

$$
\bar{A}_{l_{i} 1}^{\mathrm{T}} P_{l_{i} 11}+P_{l_{i} 11}^{\mathrm{T}} \bar{A}_{l_{i} 1}-\alpha_{l_{i}} P_{l_{i} 11}<0
$$

and $A_{l_{i} 22}^{\mathrm{T}} P_{l_{i} 22}$ is nonsingular. Therefore, $A_{l_{i} 22}$ is nonsingular, by [35] and Definition 2.2, system (6) is regular and impulse free. In the neighborhood of an equilibrium point $x(t)=$ $0, f_{\sigma(t)}(t, x(t))$ can be written as $f_{\sigma(t)}(t, x(t))=W_{\sigma(t) 0}(t) x(t)+l_{i}(t, x(t))$. Thus, system (6) can be rewritten as $E_{\sigma(t)} \dot{x}(t)=\left(\bar{A}_{\sigma(t)}+W_{\sigma(t) 0}(t)\right) x(t)+l_{i}(t, x(t))$. Then, from [38], we can obtain that

$$
W_{l_{i} 0}^{\mathrm{T}}(t) W_{l_{i} 0}(t) \leq \omega^{2} W_{l_{i}}^{\mathrm{T}} W_{l_{i}}
$$

From (14), we have

$$
\Phi_{11 l_{i}}+P_{l_{i}}^{\mathrm{T}} P_{l_{i}}+\omega^{2} W_{l_{i}}^{\mathrm{T}} W_{l_{i}}<0
$$

According to (16) and (17), we can obtain $\Phi_{11 l_{i}}+P_{l_{i}}^{\mathrm{T}} P_{l_{i}}+W_{l_{i} 0}^{\mathrm{T}}(t) W_{l_{i} 0}(t)<0$. Further, it can be obtained that

$$
\left(\bar{A}_{l_{i}}^{\mathrm{T}}+W_{l_{i} 0}^{\mathrm{T}}(t)\right) P_{l_{i}}+P_{l_{i}}^{\mathrm{T}}\left(\bar{A}_{l_{i}}+W_{l_{i} 0}(t)\right)-2 \alpha_{l_{i}} E_{l_{i}}^{\mathrm{T}} P_{l_{i}}<0 .
$$


Therefore, from the proof process of the first half, the approximation system $E_{\sigma(t)} \dot{x}(t)=$ $\left(\bar{A}_{\sigma(t)}+W_{\sigma(t) 0}(t)\right) x(t)$ is regular and impulse free. The rest of the proof is the same as in reference [38], it can be concluded that system (6) has a unique solution in the neighborhood of an equilibrium point.

Theorem 3.2 Consider the switched singular system (6), given constants $0<c_{1}<c_{2}, \alpha_{l_{i}}>0$, $\alpha_{l_{i} l_{i-1}}>0, T>0, \delta>0$, and matrix $R>0$, if there exist nonsingular matrices $P_{l_{i}}, \forall l_{i} \in \mathcal{N}$ such that (13), (14) and

$$
\begin{aligned}
& {\left[\begin{array}{ccc}
\Phi_{11 l_{i} l_{i-1}} & P_{l_{i}}^{\mathrm{T}} & W_{l_{i}}^{\mathrm{T}} \\
* & -I & 0 \\
* & * & -\gamma I
\end{array}\right]<0,} \\
& \frac{\lambda_{\min }\left(R_{1}\right) c_{2}}{\lambda_{\max }\left(\bar{R}_{1}\right) c_{1}}>\mathrm{e}^{\eta+2 \delta T},
\end{aligned}
$$

where $\Phi_{11 l_{i} l_{i-1}}=\bar{A}_{l_{i} l_{i-1}}^{\mathrm{T}} P_{l_{i}}+P_{l_{i}}^{\mathrm{T}} \bar{A}_{l_{i} l_{i-1}}-2 \alpha_{l_{i} l_{i-1}} E_{l_{i}}^{\mathrm{T}} P_{l_{i}}$ hold, then the average $d w e l l$ time of the switching signal that guarantees the regular, impulse-free nature and stability of system (6) in finite time satisfies the following formula

$$
\tau_{a l_{i}} \geq \tau_{a l_{i}}^{*}=\frac{6 \theta T}{\ln \left(\frac{\lambda_{\min }\left(R_{1}\right) c_{2}}{\lambda_{\max }\left(\bar{R}_{1}\right) c_{1}}\right)-\eta-2 \delta T},
$$

where $\eta=\sum_{k=0}^{i}\left(\alpha_{l_{k}}\left(t_{k+1}-t_{k}\right)+\left(\alpha_{l_{k} l_{k-1}}-\alpha_{l_{k}}\right) T_{l_{k} l_{k-1}}(0, t)\right)$.

Proof It remains to prove that system (1) is finite-time stabilized. By virtue of (15) and repeating the previous argument and using (19) leads to

$$
\operatorname{Re}\left(\bar{A}_{l_{i} 1}\right)<\frac{1}{2} \alpha_{l_{i}}, \quad \operatorname{Re}\left(\bar{A}_{l_{i} l_{i-1} 1}\right)<\frac{1}{2} \alpha_{l_{i} l_{i-1}} .
$$

By the definition of a matrix eigenvalue, it can be shown that there exist invertible matrices $S_{l_{i}}$ and $S_{l_{i} l_{i-1}}$ such that

$$
\begin{aligned}
& S_{l_{i}}^{-1} \bar{A}_{l_{i}} S_{l_{i}}=J\left(\bar{A}_{l_{i} 1}\right), \\
& S_{l_{i} l_{i-1}}^{-1} \bar{A}_{l_{i} l_{i-1} 1} S_{l_{i} l_{i-1}}=J\left(\bar{A}_{l_{i} l_{l_{-1} 1}}\right),
\end{aligned}
$$

where $J\left(\bar{A}_{l_{i} 1}\right), J\left(\bar{A}_{l_{i} l_{i-1} 1}\right)$ are the Jordan forms of $\bar{A}_{l_{i} 1}$ and $\bar{A}_{l_{i} l_{i-1} 1}$, respectively, $\lambda_{l_{i} 1}, \lambda_{l_{i} 2}, \ldots, \lambda_{l_{i} n}$ are the eigenvalues of the matrix $\bar{A}_{l_{i}}, \lambda_{l_{i} l_{i-1} 1}, \lambda_{l_{i} l_{i-1} 2}, \ldots, \lambda_{l_{i} l_{i-1} n}$ are the eigenvalues of the matrix $\bar{A}_{l_{i} l_{i-1} 1}$.

Combining (22) with (23), we deduce that

$$
\left\|\mathrm{e}^{\bar{A}_{l_{i}} t}\right\| \leq \mathrm{e}^{\theta_{l_{i}}+\frac{1}{2} \alpha_{l_{i}} t}, \quad\left\|\mathrm{e}^{\bar{A}_{l_{i} l_{i-1}} t}\right\| \leq \mathrm{e}^{\theta_{l_{i} l_{i-1}}+\frac{1}{2} \alpha_{l_{i} l_{i-1}} t},
$$

where $\theta_{l_{i}}=\ln \left[\lambda_{\max }\left(S_{l_{i}}\right) / \lambda_{\min }\left(S_{l_{i}}\right)\right], \theta_{l_{i} l_{i-1}}=\ln \left[\lambda_{\max }\left(S_{l_{i} l_{i-1}}\right) / \lambda_{\min }\left(S_{l_{i} l_{i-1}}\right)\right]$, use $\lambda_{\max }\left(S_{l_{i}}\right)$ to represent the maximum eigenvalue of matrix $S_{l_{i}}, \lambda_{\max }\left(S_{l_{i} l_{i-1}}\right)$ denotes the maximum of all eigenvalues of matrix $S_{l_{i} l_{i-1}}$. 
Denoting $\max _{l_{i}, l_{i-1} \in \mathcal{N}}\left\{\theta_{l_{i}}, \theta_{l_{i} l_{i-1}}\right\}$ briefly by $\theta$ with the notation $\alpha=\max _{l_{i}, l_{i-1} \in \mathcal{N}}\left\{\alpha_{l_{i}}, \alpha_{l_{i} l_{i-1}}\right\}$ and using equation (24), we obtain

$$
\left\|\mathrm{e}^{\bar{A}_{l_{i} 1} t}\right\| \leq \mathrm{e}^{\theta+\frac{1}{2} \alpha_{l_{i}} t}, \quad\left\|\mathrm{e}^{\bar{A}_{l_{i} l_{i-1} 1 t}}\right\| \leq \mathrm{e}^{\theta+\frac{1}{2} \alpha_{l_{i} l_{i-1}} t}, \quad \forall t \geq 0
$$

For any $t \in\left[t_{0}, t_{1}\right)$, according to the theoretical knowledge of the solution of the differential equation, when the initial state satisfies $x_{1}\left(t_{0}^{-}\right)=x_{1}(0)$, the solution of equation (6) is

$$
\bar{x}_{1}(t)=\mathrm{e}^{\bar{A}_{\sigma\left(t_{0}\right) 1} t} x_{1}\left(t_{0}^{-}\right)+\int_{0}^{t} \mathrm{e}^{\bar{A}_{\sigma\left(t_{0}\right) 1}(t-s)} h_{\sigma\left(t_{0}\right) 1}(s) \mathrm{d} s .
$$

Furthermore, for any $t \in\left[t_{1}, \tilde{t}_{1}\right)$ and noting $x_{1}\left(t_{1}\right)=x_{1}\left(t_{1}^{-}\right)$, combined with (26), yields

$$
\begin{aligned}
\bar{x}_{1}(t)= & \mathrm{e}^{\bar{A}_{\sigma\left(t_{1}\right) \sigma\left(t_{0}\right) 1}\left(t-t_{1}\right)} x_{1}\left(t_{1}\right)+\int_{t_{1}}^{t} \mathrm{e}^{\bar{A}_{\sigma\left(t_{1}\right) \sigma\left(t_{0}\right) 1}(t-s)} h_{\sigma\left(t_{1}\right) 1}(s) \mathrm{d} s \\
= & \mathrm{e}^{\bar{A}_{\sigma\left(t_{1}\right) \sigma\left(t_{0}\right) 1}\left(t-t_{1}\right)}\left[\mathrm{e}^{\bar{A}_{\sigma\left(t_{0}\right) 1} t_{1}} x_{1}(0)+\int_{0}^{t_{1}} \mathrm{e}^{\bar{A}_{\sigma\left(t_{0}\right) 1}(t-s)} h_{\sigma\left(t_{0}\right) 1}(s) \mathrm{d} s\right] \\
& +\int_{t_{1}}^{t} \mathrm{e}^{\bar{A}_{\sigma\left(t_{1}\right) \sigma\left(t_{0}\right) 1}(t-s)} h_{\sigma\left(t_{1}\right) 1}(s) \mathrm{d} s .
\end{aligned}
$$

Similarly, $x_{1}\left(\tilde{t}_{1}\right)=x_{1}\left(\tilde{t}_{1}^{-}\right)$holds, and we have that for any $t \in\left[\tilde{t}_{1}, t_{2}\right)$

$$
\begin{aligned}
\bar{x}_{1}(t)= & \mathrm{e}^{\bar{A}_{\sigma\left(t_{1}\right) 1}\left(t-\tilde{t}_{1}\right)} x_{1}\left(\tilde{t}_{1}\right)+\int_{\tilde{t}_{1}}^{t} \mathrm{e}^{\bar{A}_{\sigma\left(t_{1}\right) 1}(t-s)} h_{\sigma\left(t_{1}\right) 1}(s) \mathrm{d} s \\
= & \mathrm{e}^{\bar{A}_{\sigma\left(t_{1}\right) 1}\left(t-\tilde{t}_{1}\right)}\left[\mathrm{e}^{\bar{A}_{\sigma\left(t_{1}\right) \sigma\left(t_{0}\right) 1} \tau\left(t_{1}\right)}\left[\mathrm{e}^{\bar{A}_{\sigma\left(t_{0}\right) 1} t_{1}} x_{1}(0)+\int_{0}^{t_{1}} \mathrm{e}^{\bar{A}_{\sigma\left(t_{0}\right) 1}(t-s)} h_{\sigma\left(t_{0}\right) 1}(s) \mathrm{d} s\right]\right. \\
& \left.+\int_{t_{1}}^{\tilde{t}_{1}} \mathrm{e}^{\bar{A}_{\sigma\left(t_{1}\right) \sigma\left(t_{0}\right) 1}(t-s)} h_{\sigma\left(t_{1}\right) 1}(s) \mathrm{d} s\right]+\int_{\tilde{t}_{1}}^{t} \mathrm{e}^{\bar{A}_{\sigma\left(t_{1}\right) \sigma\left(t_{0}\right) 1}(t-s)} h_{\sigma\left(t_{1}\right) 1}(s) \mathrm{d} s \\
= & \mathrm{e}^{\bar{A}_{\sigma\left(t_{1}\right) 1}\left(t-\tilde{t}_{1}\right)} \mathrm{e}^{\bar{A}_{\sigma\left(t_{1}\right) \sigma\left(t_{0}\right) 1} \tau\left(t_{1}\right)} \mathrm{e}^{\bar{A}_{\sigma\left(t_{0}\right) 1} t_{1}} x_{1}(0) \\
& +\mathrm{e}^{\bar{A}_{\sigma\left(t_{1}\right) 1}\left(t-\tilde{t}_{1}\right)} \mathrm{e}^{\bar{A}_{\sigma\left(t_{1}\right) \sigma\left(t_{0}\right) 1} \tau\left(t_{1}\right)} \int_{0}^{t_{1}} \mathrm{e}^{\bar{A}_{\sigma\left(t_{0}\right) 1}(t-s)} h_{\sigma\left(t_{0}\right) 1}(s) \mathrm{d} s \\
& +\mathrm{e}^{\bar{A}_{\sigma\left(t_{1}\right) 1}\left(t-\tilde{t}_{1}\right)} \int_{t_{1}}^{\tilde{t}_{1}} \mathrm{e}^{\bar{A}_{\sigma\left(t_{1}\right) \sigma\left(t_{0}\right) 1}(t-s)} h_{\sigma\left(t_{1}\right) 1}(s) \mathrm{d} s \\
& +\int_{\tilde{t}_{1}}^{t} \mathrm{e}^{\bar{A}_{\sigma\left(t_{1}\right) \sigma\left(t_{0}\right) 1}(t-s)} h_{\sigma\left(t_{1}\right) 1}(s) \mathrm{d} s .
\end{aligned}
$$

Under the conditions stated above, when $t \in\left[t_{i}, \tilde{t}_{i}\right)$, we infer that

$$
\begin{aligned}
\bar{x}_{1}(t)= & \mathrm{e}^{\bar{A}_{\sigma\left(t_{i}\right) \sigma\left(t_{i-1}\right) 1}\left(t-t_{i}\right)} \mathrm{e}^{\bar{A}_{\sigma\left(t_{i-1}\right) 1}\left(t_{i}-\tilde{t}_{i-1}\right)} \ldots \mathrm{e}^{\bar{A}_{\sigma\left(t_{0}\right) 1} t_{1}} x_{1}(0) \\
& +\int_{0}^{t_{1}} \mathrm{e}^{\bar{A}_{\sigma\left(t_{i}\right) \sigma\left(t_{i-1}\right) 1}\left(t-t_{i}\right)} \mathrm{e}^{\bar{A}_{\sigma\left(t_{i-1}\right) 1}\left(t_{i}-\tilde{t}_{i-1}\right)} \ldots \mathrm{e}^{\bar{A}_{\sigma\left(t_{0}\right) 1}\left(t_{1}-s\right)} h_{\sigma\left(t_{0}\right) 1}(s) \mathrm{d} s \\
& +\int_{t_{1}}^{\tilde{t}_{1}} \mathrm{e}^{\bar{A}_{\sigma\left(t_{i}\right) \sigma\left(t_{i-1}\right) 1}\left(t-t_{i}\right)} \mathrm{e}^{\bar{A}_{\sigma\left(t_{i-1}\right) 1}\left(t_{i}-\tilde{t}_{i-1}\right)} \ldots \mathrm{e}^{\bar{A}_{\sigma\left(t_{1}\right) \sigma\left(t_{0}\right) 1}\left(\tilde{t}_{1}-s\right)} h_{\sigma\left(t_{1}\right) 1}(s) \mathrm{d} s
\end{aligned}
$$




$$
\begin{aligned}
& +\cdots+\int_{\tilde{t}_{i-1}}^{t_{i}} \mathrm{e}^{\bar{A}_{\sigma\left(t_{i}\right) \sigma\left(t_{i-1}\right) 1}\left(t-t_{i}\right)} \mathrm{e}^{\bar{A}_{\sigma\left(t_{i-1}\right) 1}\left(t_{i}-s\right)} h_{\sigma\left(t_{i-1}\right) 1}(s) \mathrm{d} s \\
& +\int_{t_{i}}^{t} \mathrm{e}^{\bar{A}_{\sigma\left(t_{i}\right) \sigma\left(t_{i-1}\right) 1}(t-s)} h_{\sigma\left(t_{i}\right) 1}(s) \mathrm{d} s .
\end{aligned}
$$

Thus, $\left\|\bar{x}_{1}(t)\right\|$ can be bounded by

$$
\begin{aligned}
\left\|\bar{x}_{1}(t)\right\| \leq & \mathrm{e}^{\theta+\frac{1}{2} \alpha{ }_{l_{i} l_{i-1}}\left(t-t_{i}\right)} \mathrm{e}^{\theta+\frac{1}{2} \alpha l_{i-1}\left(t_{i}-\tilde{t}_{i-1}\right)} \ldots \mathrm{e}^{\theta+\frac{1}{2} \alpha l_{0} t_{1}}\left\|x_{1}(0)\right\| \\
& +\int_{0}^{t_{1}} \mathrm{e}^{\theta+\frac{1}{2} \alpha l_{l_{i} l_{i-1}}\left(t-t_{i}\right)} \mathrm{e}^{\theta+\frac{1}{2} \alpha l_{l_{i-1}}\left(t_{i}-\tilde{t}_{i-1}\right)} \ldots \mathrm{e}^{\theta+\frac{1}{2} \alpha l_{0}\left(t_{1}-s\right)}\left\|h_{\sigma\left(t_{0}\right) 1}(s)\right\| \mathrm{d} s \\
& +\int_{t_{1}}^{\tilde{t}_{1}} \mathrm{e}^{\theta+\frac{1}{2} \alpha l_{i} l_{i-1}\left(t-t_{i}\right)} \mathrm{e}^{\theta+\frac{1}{2} \alpha l_{i-1}\left(t_{i}-\tilde{t}_{i-1}\right)} \ldots \mathrm{e}^{\theta+\frac{1}{2} \alpha l_{1} l_{0}\left(\tilde{t}_{1}-s\right)}\left\|h_{\sigma\left(t_{1}\right) 1}(s)\right\| \mathrm{d} s \\
& +\cdots+\int_{\tilde{t}_{i-1}}^{t_{i}} \mathrm{e}^{\theta+\frac{1}{2} \alpha l_{i} l_{i-1}\left(t-t_{i}\right)} \mathrm{e}^{\theta+\frac{1}{2} \alpha l_{i-1}\left(t_{i}-s\right)}\left\|h_{\sigma\left(t_{i-1}\right) 1}(s)\right\| \mathrm{d} s \\
& +\int_{t_{i}}^{t} \mathrm{e}^{\theta+\frac{1}{2} \alpha l_{i} l_{i-1}(t-s)}\left\|h_{\sigma\left(t_{k}\right) 1}(s)\right\| \mathrm{d} s .
\end{aligned}
$$

Using (12), (30) shows that

$$
\begin{aligned}
\left\|\bar{x}_{1}(t)\right\| \leq & \mathrm{e}^{2 N_{\sigma}(0, t) \theta+\frac{1}{2} \sum_{k=0}^{i}\left(\alpha_{l_{k}} T_{l_{k}}(0, t)+\alpha l_{k} l_{k-1} T_{l_{k} l_{k-1}}(0, t)\right)}\left\|x_{1}(0)\right\| \\
& +\int_{0}^{t} \mathrm{e}^{\left(2 N_{\sigma}(s, t)+1\right) \theta+\frac{1}{2} \sum_{k=0}^{i}\left(\alpha_{l_{k}} T_{l_{k}}(s, t)+\alpha l_{k} l_{k-1} T_{l_{k} l_{k-1}}(s, t)\right)} \delta\left\|\bar{x}_{1}(s)\right\| \mathrm{d} s .
\end{aligned}
$$

Moreover, for $t \in\left[\tilde{t}_{i}, t_{i+1}\right)$,

$$
\begin{aligned}
\bar{x}_{1}(t)= & \mathrm{e}^{\bar{A}_{\sigma\left(t_{i}\right) 1}\left(t-\tilde{t}_{i}\right)} \mathrm{e}^{\bar{A}_{\sigma\left(t_{i}\right) \sigma\left(t_{i-1}\right) 1} \tau\left(t_{i}\right)} \ldots \mathrm{e}^{\bar{A}_{\sigma\left(t_{0}\right) 1} t_{1}} x_{1}(0) \\
& +\int_{0}^{t_{1}} \mathrm{e}^{\bar{A}_{\sigma\left(t_{i}\right) 1}\left(t-\tilde{t}_{i}\right)} \mathrm{e}^{\bar{A}_{\sigma\left(t_{i}\right) \sigma\left(t_{i-1}\right) 1} \tau\left(t_{i}\right)} \ldots \mathrm{e}^{\bar{A}_{\sigma\left(t_{0}\right) 1}\left(t_{1}-s\right)} h_{\sigma\left(t_{0}\right) 1}(s) \mathrm{d} s \\
& +\int_{t_{1}}^{\tilde{t}_{1}} \mathrm{e}^{\bar{A}_{\sigma\left(t_{i}\right) 1}\left(t-\tilde{t}_{i}\right)} \mathrm{e}^{\bar{A}_{\sigma\left(t_{i}\right) \sigma\left(t_{i-1}\right) 1} \tau\left(t_{i}\right)} \ldots \mathrm{e}^{\bar{A}_{\sigma\left(t_{i}\right) \sigma\left(t_{i-1}\right) 1}\left(\tilde{t}_{1}-s\right)} h_{\sigma\left(t_{1}\right) 1}(s) \mathrm{d} s \\
& +\cdots+\int_{\tilde{t}_{i-1}}^{t_{i}} \mathrm{e}^{\bar{A}_{\sigma\left(t_{i}\right) 1}\left(t-\tilde{t}_{i}\right)} \mathrm{e}^{\bar{A}_{\sigma\left(t_{i}\right) \sigma\left(t_{i-1}\right) 1}\left(\tilde{t}_{i}-s\right)} h_{\sigma\left(t_{i-1}\right) 1}(s) \mathrm{d} s \\
& +\int_{t_{i}}^{t} \mathrm{e}^{\bar{A}_{\sigma\left(t_{i}\right) 1}(t-s)} h_{\sigma\left(t_{i}\right) 1}(s) \mathrm{d} s .
\end{aligned}
$$

Accordingly, we can obtain

$$
\begin{aligned}
\left\|\bar{x}_{1}(t)\right\| \leq & \mathrm{e}^{\theta+\frac{1}{2} \alpha l_{i}\left(t-\tilde{t}_{i}\right)} \mathrm{e}^{\theta+\frac{1}{2} \alpha l_{l_{i} l_{i-1}}\left(\tilde{t}_{i}-t_{i}\right)} \ldots \mathrm{e}^{\theta+\frac{1}{2} \alpha l_{0} t_{1}}\left\|x_{1}(0)\right\| \\
& +\int_{0}^{t_{1}} \mathrm{e}^{\theta+\frac{1}{2} \alpha_{l_{i}}\left(t-\tilde{t}_{i}\right)} \mathrm{e}^{\theta+\frac{1}{2} \alpha l_{l_{i} l_{i-1}}\left(\tilde{t}_{i}-t_{i}\right)} \ldots \mathrm{e}^{\theta+\frac{1}{2} \alpha l_{0}\left(t_{1}-s\right)}\left\|h_{\sigma\left(t_{0}\right) 1}(s)\right\| \mathrm{d} s \\
& +\int_{t_{1}}^{\tilde{t}_{1}} \mathrm{e}^{\theta+\frac{1}{2} \alpha_{l_{i}}\left(t-\tilde{t}_{i}\right)} \mathrm{e}^{\theta+\frac{1}{2} \alpha l_{l_{i} l_{i-1}}\left(\tilde{t}_{i}-t_{i}\right)} \ldots \mathrm{e}^{\theta+\frac{1}{2} \alpha l_{1} l_{0}\left(\tilde{t}_{1}-s\right)}\left\|h_{\sigma\left(t_{1}\right) 1}(s)\right\| \mathrm{d} s \\
& +\cdots+\int_{\tilde{t}_{i-1}}^{t_{i}} \mathrm{e}^{\theta+\frac{1}{2} \alpha l_{i}\left(t-\tilde{t}_{i}\right)} \mathrm{e}^{\theta+\frac{1}{2} \alpha_{l_{i} l_{i-1}}\left(\tilde{t}_{i}-s\right)}\left\|h_{\sigma\left(t_{i-1}\right) 1}(s)\right\| \mathrm{d} s
\end{aligned}
$$




$$
+\int_{t_{i}}^{t} \mathrm{e}^{\theta+\frac{1}{2} \alpha l_{i}(t-s)}\left\|h_{\sigma\left(t_{i}\right) 1}(s)\right\| \mathrm{d} s
$$

Combining (12) and (33) leads to

$$
\begin{aligned}
\left\|\bar{x}_{1}(t)\right\| \leq & \mathrm{e}^{\left(2 N_{\sigma}(0, t)+1\right) \theta+\frac{1}{2} \sum_{k=0}^{i}\left(\alpha_{l_{k}} T_{l_{k}}(0, t)+\alpha_{l_{k}} l_{k-1} T_{l_{k} l_{k-1}}(0, t)\right)}\left\|x_{1}(0)\right\| \\
& +\int_{0}^{t} \mathrm{e}^{\left(2 N_{\sigma}(s, t)+1\right) \theta+\frac{1}{2} \sum_{k=0}^{i}\left(\alpha_{l_{k}} T_{l_{k}}(s, t)+\alpha_{l_{k}} l_{k-1} T_{l_{k}} l_{k-1}(s, t)\right)} \delta\left\|\bar{x}_{1}(s)\right\| \mathrm{d} s .
\end{aligned}
$$

On account of the above discussion, formula (34) holds for any $t \in\left[t_{i}, t_{i+1}\right)$. Since $1 \leq$ $N_{\sigma}(0, t) \leq \frac{t}{\tau_{a}}$, we have

$$
\begin{aligned}
\left\|\bar{x}_{1}(t)\right\| \leq & \mathrm{e}^{\left(\frac{2 t}{\tau_{a}}+1\right) \theta+\frac{1}{2} \sum_{k=0}^{i}\left(\alpha_{l_{k}} T_{l_{k}}(0, t)+\alpha l_{k} l_{k-1} T_{l_{k} l_{k-1}}(0, t)\right)}\left\|x_{1}(0)\right\| \\
& +\int_{0}^{t} \mathrm{e}^{\left(\frac{2(t-s)}{\tau a}+1\right) \theta+\frac{1}{2} \sum_{k=0}^{i}\left(\alpha_{l_{k}} T_{l_{k}}(s, t)+\alpha_{l_{k}} l_{k-1} T_{l_{k} l_{k-1}}(s, t)\right)} \delta\left\|\bar{x}_{1}(s)\right\| \mathrm{d} s \\
\leq & \mathrm{e}^{\frac{3 t}{\tau_{a}} \theta+\frac{1}{2} \sum_{k=0}^{i}\left(\alpha l_{k} T_{l_{k}}(0, t)+\alpha l_{k} l_{k-1} T_{l_{k} l_{k-1}}(0, t)\right)}\left\|x_{1}(0)\right\| \\
& +\int_{0}^{t} \mathrm{e}^{\frac{3(t-s)}{\tau a} \theta+\frac{1}{2} \sum_{k=0}^{i}\left(\alpha l_{k} T_{l_{k}}(s, t)+\alpha l_{k} l_{k-1} T_{l_{k} l_{k-1}}(s, t)\right)} \delta\left\|\bar{x}_{1}(s)\right\| \mathrm{d} s .
\end{aligned}
$$

On multiplying both sides of (35) by $\mathrm{e}^{-\frac{3 t}{\tau a} \theta-\frac{1}{2} \sum_{k=0}^{i}\left(\alpha_{l_{k}} T_{l_{k}}(0, t)+\alpha_{l_{k}} l_{k-1} T_{l_{k} l_{k-1}}(0, t)\right)}$, we obtain

$$
\begin{aligned}
& \mathrm{e}^{-\frac{3 t \theta}{\tau_{a}}-\frac{1}{2} \sum_{k=0}^{i}\left(\alpha_{l_{k}} T_{l_{k}}(0, t)+\alpha l_{k} l_{k-1} T_{l_{k} l_{k-1}}(0, t)\right)}\left\|\bar{x}_{1}(t)\right\| \\
& \quad \leq\left\|\bar{x}_{1}(0)\right\|+\int_{0}^{t} \mathrm{e}^{-\frac{3 s \theta}{\tau_{a}}-\frac{1}{2} \sum_{k=0}^{i}\left(\alpha_{l_{k}} T_{l_{k}}(0, s)+\alpha l_{k} l_{k-1} T_{l_{k} l_{k-1}}(0, s)\right)} \delta\left\|\bar{x}_{1}(s)\right\| \mathrm{d} s .
\end{aligned}
$$

Then, it can be deduced from Lemma 2.2 that

$$
\mathrm{e}^{-\frac{3 t \theta}{\tau_{a}}-\frac{1}{2} \sum_{k=0}^{i}\left(\alpha_{l_{k}} T_{l_{k}}(0, t)+\alpha l_{k} l_{k-1} T_{l_{k} l_{k-1}}(0, t)\right)}\left\|\bar{x}_{1}(t)\right\| \leq\left\|\bar{x}_{1}(0)\right\| \mathrm{e}^{\delta t} .
$$

That is,

$$
\left\|\bar{x}_{1}(t)\right\| \leq \mathrm{e}^{\left(\frac{3 \theta}{\tau_{a}}+\delta\right) t+\frac{1}{2} \sum_{k=0}^{i}\left(\alpha_{l_{k}} T_{l_{k}}(0, t)+\alpha l_{k} l_{k-1} T_{l_{k} l_{k-1}}(0, t)\right)}\left\|\bar{x}_{1}(0)\right\| .
$$

Using the expressions of

$$
M_{\sigma(0)}^{-\mathrm{T}} R M_{\sigma(0)}^{-1}=\left[\begin{array}{ll}
R_{1} & R_{2} \\
R_{3} & R_{4}
\end{array}\right], \quad M_{\sigma(t)}^{-\mathrm{T}} R M_{\sigma(t)}^{-1}=\left[\begin{array}{cc}
\bar{R}_{1} & \bar{R}_{2} \\
\bar{R}_{3} & \bar{R}_{4}
\end{array}\right] .
$$

Noting that $x(t)=N_{\sigma(t)} \bar{x}(t)$, we can show that

$$
\begin{aligned}
x^{\mathrm{T}} & (0) E_{\sigma(0)}^{\mathrm{T}} R E_{\sigma(0)} x(0) \\
& =x^{\mathrm{T}}(0) N_{\sigma(0)}^{-\mathrm{T}} N_{\sigma(0)}^{\mathrm{T}} E_{\sigma(0)}^{\mathrm{T}} M_{\sigma(0)}^{\mathrm{T}} M_{\sigma(0)}^{-\mathrm{T}} R M_{\sigma(0)}^{-1} M_{\sigma(0)} E_{\sigma(0)} N_{\sigma(0)} N_{\sigma(0)}^{-1} x(0) \\
& =\left[\begin{array}{ll}
\bar{x}_{1}^{\mathrm{T}}(0) & \bar{x}_{2}^{\mathrm{T}}(0)
\end{array}\right]\left[\begin{array}{rr}
I_{r} & 0 \\
0 & 0
\end{array}\right]\left[\begin{array}{ll}
R_{1} & R_{2} \\
R_{3} & R_{4}
\end{array}\right]\left[\begin{array}{cc}
I_{r} & 0 \\
0 & 0
\end{array}\right]\left[\begin{array}{c}
\bar{x}_{1}(0) \\
\bar{x}_{2}(0)
\end{array}\right] \\
& =\bar{x}_{1}^{\mathrm{T}}(0) R_{1} \bar{x}_{1}(0) \leq c_{1} .
\end{aligned}
$$


Similarly, $x^{\mathrm{T}}(t) E_{\sigma(t)}^{\mathrm{T}} R E_{\sigma(t)} x(t) \leq c_{2}$ can be derived from $\bar{x}_{1}^{\mathrm{T}}(t) \bar{R}_{1} \bar{x}_{1}(t) \leq c_{2}$. It follows from (39) that $\left\|\bar{x}_{1}(0)\right\|^{2} \leq \frac{c_{1}}{\lambda_{\min }\left(R_{1}\right)}$. By switching signal (21) and (37), it holds that

$$
\begin{aligned}
\bar{x}_{1}^{\mathrm{T}}(t) \bar{R}_{1} \bar{x}_{1}(t) & \leq \lambda_{\max }\left(\bar{R}_{1}\right) \bar{x}_{1}^{\mathrm{T}}(t) \bar{x}_{1}(t) \\
& \leq \lambda_{\max }\left(\bar{R}_{1}\right) \mathrm{e}^{2 T\left(\frac{3 \theta}{\tau_{a}}+\delta\right)+\sum_{k=0}^{i}\left(\alpha_{l_{k}} T_{l_{k}}(0, t)+\alpha_{l_{k}} l_{k-1} T_{l_{k} l_{k-1}}(0, t)\right)} \frac{c_{1}}{\lambda_{\min }\left(R_{1}\right)} \\
& =\mathrm{e}^{2 T\left(\frac{3 \theta}{\tau_{a}}+\delta\right)+\sum_{k=0}^{i}\left(\alpha_{l_{k}}\left(t_{k+1}-t_{k}\right)+\left(\alpha_{l_{k} l_{k-1}}-\alpha_{l_{k}}\right) T_{l_{k} l_{k-1}}(0, t)\right)} \frac{c_{1} \lambda_{\max }\left(\bar{R}_{1}\right)}{\lambda_{\min }\left(R_{1}\right)} \\
& \leq c_{2} .
\end{aligned}
$$

Thus, the proof is completed.

Remark 3.3 In reference [24], the authors' proof is given on the basis of assuming that the subsystem is regular, impulse free and stable. The proof in this paper removes this hypothesis and proves the regularity and nonpulsation of the switching system through the known constraints. It is worth noting that the matrix $E_{\sigma(t)}$ in this paper varies with different subsystems, so the conclusion has stronger applicability. When $E_{\sigma(t)}$ is a nonsingular matrix, the system studied in this paper degenerates into a general switching system.

Remark 3.4 In the proof of the above theorem, no Lyapunov function is constructed. Instead, starting with the state-equation solution of the switched system with nonlinear disturbance and taking the switching time point as the boundary, the operation time period of each switched system is analyzed. The state solutions of the closed-loop system in the matched time period and the mismatched time period are given, and the state solutions of the whole time period are obtained by alternating iterative derivation. The condition that all subsystems must be stable is removed.

Theorem 3.3 Consider the switched singular system (6), given constants $0<c_{1}<c_{2}, \alpha>0$, $T>0, \delta>0$, matrix $R>0$ and a full column rank matrix $X_{l_{i}} \in \mathbb{R}^{n \times(n-r)}$ satisfies $E_{l_{i}} X_{l_{i}}=0$, if there exist matrices $F_{l_{i}}>0, S_{l_{i}}, G_{l_{i}}, \forall l_{i} \in \mathcal{N}$ such that (20) and the following LMIs hold

$$
\begin{aligned}
& {\left[\begin{array}{ccc}
\Gamma_{11 l_{i}} & I & \Omega_{l_{i}}^{\mathrm{T}} W_{l_{i}}^{\mathrm{T}} \\
* & -I & 0 \\
* & * & -\gamma I
\end{array}\right]<0,} \\
& {\left[\begin{array}{ccc}
\Gamma_{11 l_{i} l_{i-1}} & I & \Omega_{l_{i}}^{\mathrm{T}} W_{l_{i}}^{\mathrm{T}} \\
* & -I & 0 \\
* & * & -\gamma I
\end{array}\right]<0 .}
\end{aligned}
$$

where $\Gamma_{11 l_{i}}=A_{l_{i}} \Omega_{l_{i}}+B_{l_{i}} G_{l_{i}}+\Omega_{l_{i}}^{\mathrm{T}} A_{l_{i}}^{\mathrm{T}}+G_{l_{i}}^{\mathrm{T}} B_{l_{i}}^{\mathrm{T}}-2 \alpha_{l_{i}} \Omega_{l_{i}}^{\mathrm{T}} E_{l_{i}}^{\mathrm{T}}, \Gamma_{11 l_{i} l_{i-1}}=A_{l_{i}} \Omega_{l_{i}}+B_{l_{i}} G_{l_{i} l_{i-1}}+\Omega_{l_{i}}^{\mathrm{T}} A_{l_{i}}^{\mathrm{T}}+$ $G_{l_{i} l_{i-1}}^{\mathrm{T}} B_{l_{i}}^{\mathrm{T}}-2 \alpha_{l_{i} l_{i-1}} \Omega_{l_{i}}^{\mathrm{T}} E_{l_{i}}^{\mathrm{T}}$. Then, the average dwell time of the switching signal that guarantees the finite-time stabilization of system (6) satisfies (21). Moreover, the controller gain is given by

$$
\begin{aligned}
& u(t)=G_{l_{i}} \Omega_{l_{i}}^{-1} x(t), \\
& \Omega_{l_{i}}=F_{l_{i}} E_{l_{i}}^{\mathrm{T}}+X_{l_{i}} S_{l_{i}} .
\end{aligned}
$$


Proof In order to obtain the controller gain, we denote $D_{l_{i}}^{\mathrm{T}}=P_{l_{i}}^{-\mathrm{T}}, D_{l_{i}}=P_{l_{i}}^{-1}$. From (5), we have $\bar{A}_{l_{i}}=A_{l_{i}}+B_{l_{i}} K_{l_{i}}, \bar{A}_{l_{i} l_{i-1}}=A_{l_{i}}+B_{l_{i}} K_{l_{i-1}}$. Pre- and postmultiplying (14) and (19) by $\operatorname{diag}\left\{D_{l_{i}}^{\mathrm{T}}, I, I\right\}$ and its transpose, respectively, and using the definition of $G_{l_{i}}=K_{l_{i}} D_{l_{i}}$, it follows that

$$
\left[\begin{array}{ccc}
\Sigma_{11 l_{i}} & I & D_{l_{i}}^{\mathrm{T}} W_{l_{i}}^{\mathrm{T}} \\
* & -I & 0 \\
* & * & -\gamma I
\end{array}\right]<0,
$$

where $\Sigma_{11 l_{i}}=D_{l_{i}}^{\mathrm{T}} A_{l_{i}}^{\mathrm{T}}+G_{l_{i}}^{\mathrm{T}} B_{l_{i}}^{\mathrm{T}}+A_{l_{i}} D_{l_{i}}+B_{l_{i}} G_{l_{i}}-2 \alpha_{l_{i}} D_{l_{i}}^{\mathrm{T}} E_{l_{i}}^{\mathrm{T}}$. Similarly, we obtain

$$
\left[\begin{array}{ccc}
\Sigma_{11 l_{i} l_{i-1}} & I & D_{l_{i}}^{\mathrm{T}} W_{l_{i}}^{\mathrm{T}} \\
* & -I & 0 \\
* & * & -\gamma I
\end{array}\right]<0,
$$

where $\Sigma_{1 l_{i} l_{i-1}}=D_{l_{i}}^{\mathrm{T}} A_{l_{i}}^{\mathrm{T}}+G_{l_{i} l_{i-1}}^{\mathrm{T}} B_{l_{i}}^{\mathrm{T}}+A_{l_{i}} D_{l_{i}}+B_{l_{i}} G_{l_{i} l_{i-1}}-2 \alpha_{l_{i} l_{i-1}} D_{l_{i}}^{\mathrm{T}} E_{l_{i}}^{\mathrm{T}}$. Substituting $D_{l_{i}}=\Omega_{l_{i}}=$ $F_{l_{i}} E^{\mathrm{T}}+X_{l_{i}} S_{l_{i}}$ into (44) and (45), respectively, and denoting $G_{l_{i}}=K_{l_{i}} \Omega_{l_{i}},(41)((42))$ is equivalent to $(44)((45))$.

Remark 3.5 The proof of the theorem does not require the stability of the subsystem and parameter $\alpha$ can take different values $\alpha_{l_{i}}$ for different subsystems so it is less conservative. Compared with Theorem 3.1 in [25], the constraint conditions of equations (17) and (18) are discarded. The subsystem and the corresponding controller are one-to-one corresponding. Therefore, the design of the controller is only related to the subscript $l_{i}$ and not dependent on $l_{i-1}$.

When the switching delay is not considered, that is, when the operation of the controller and the corresponding subsystem is synchronous, we can obtain the following corollary. It is worth noting that the controller of the system becomes

$$
u(t)=K_{\sigma(t)} x(t) .
$$

Corollary 3.1 Consider the switched singular system (6) with control input (46), given constants $0<c_{1}<c_{2}, \alpha>0, T>0, \delta>0$, matrix $R>0$ and a full column rank matrix $X_{l_{i}} \in \mathbb{R}^{n \times(n-r)}$ satisfies $E_{l_{i}} X_{l_{i}}=0$, if there exist matrices $F_{l_{i}}>0, S_{l_{i}}, G_{l_{i}}, \forall l_{i} \in \mathcal{N}$ such that

$$
\frac{\lambda_{\min }\left(R_{1}\right) c_{2}}{\lambda_{\max }\left(\bar{R}_{1}\right) c_{1}}>(\alpha+2 \delta) T
$$

and (41) hold. Then, the average dwell time of the switching signal that guarantees the finite-time stabilization of system (6) with respect to $\left(c_{1}, c_{2}, T, R, \sigma\right)$ satisfies

$$
\tau_{a l_{i}} \geq \tau_{a l_{i}}^{*}=\frac{6 \theta T}{\ln \left(\frac{\lambda_{\min }\left(R_{1}\right) c_{2}}{\lambda_{\max }\left(R_{1}\right) c_{1}}\right)-(\alpha+2 \delta) T},
$$

where $\alpha=\max _{l_{i}, l_{i-1} \in \mathcal{N}}\left\{\alpha_{l_{i}}, \alpha_{l_{i} l_{i-1}}\right\}$. Moreover, the controller gain is given by (43). 


\section{Numerical example}

In this section, two numerical examples are provided to demonstrate the validity and feasibility of the above results.

Example 1 Consider the switched nonlinear singular system (1) with two subsystems and matrix parameters as follows:

Subsystem1:

$$
\begin{aligned}
& E_{1}=\left[\begin{array}{lll}
1 & 1 & 1 \\
1 & 0 & 1 \\
0 & 0 & 0
\end{array}\right], \quad A_{1}=\left[\begin{array}{ccc}
-0.7 & -2.6 & 1 \\
-1.5 & 0 & -1.5 \\
1 & -1 & -2
\end{array}\right], \\
& B_{1}=\left[\begin{array}{ll}
0 & 0 \\
1 & 2 \\
0 & 1
\end{array}\right], \quad f_{1}=\left[\begin{array}{c}
0.1 \sin \left(x_{1}(t)\right) \\
0.1 \sin \left(x_{2}(t)\right) \\
0
\end{array}\right],
\end{aligned}
$$

Subsystem2:

$$
\begin{aligned}
& E_{2}=\left[\begin{array}{lll}
1 & 0 & 1 \\
0 & 1 & 0 \\
0 & 0 & 0
\end{array}\right], \quad A_{2}=\left[\begin{array}{ccc}
-1.7 & 0 & -1.5 \\
0.8 & -2.1 & 2.2 \\
3 & -0.6 & -1
\end{array}\right], \\
& B_{2}=\left[\begin{array}{ll}
1 & 0 \\
1 & 2 \\
0 & 1
\end{array}\right], \quad f_{2}=\left[\begin{array}{c}
\sin \left(0.1 x_{1}(t)\right) \\
0 \\
\cos \left(x_{2}(t)\right)
\end{array}\right] .
\end{aligned}
$$

Choosing two sets of matrices as follows that can transform matrices $E_{1}$ and $E_{2}$ into a unit matrix, respectively. Selecting $X_{1}, X_{2}$ that satisfy equation $E X_{1}=E X_{2}=0$ as follows

$$
\begin{aligned}
& M_{1}\left[\begin{array}{ccc}
0 & 1 & 0 \\
1 & -1 & 0 \\
0 & 0 & 1
\end{array}\right], \quad N_{1}=\left[\begin{array}{ccc}
1 & 0 & -1 \\
0 & 1 & 0 \\
0 & 0 & 1
\end{array}\right], \quad M_{2}=I_{3}, \\
& N_{2}=\left[\begin{array}{ccc}
1 & 0 & -1 \\
0 & 1 & 0 \\
0 & 0 & 1
\end{array}\right], \quad X_{1}=X_{2}=\left[\begin{array}{c}
-1 \\
0 \\
1
\end{array}\right], \\
& R=I, \quad M_{1}^{-\mathrm{T}} R M_{1}^{-1}=\left[\begin{array}{lll}
2 & 1 & 0 \\
1 & 1 & 0 \\
0 & 0 & 1
\end{array}\right], \quad M_{2}^{-\mathrm{T}} R M_{2}^{-1}=I_{3} .
\end{aligned}
$$

Suppose $W_{1}=W_{2}=\left[\begin{array}{lll}1 & 1 & 1\end{array}\right], \omega=1, \alpha_{1}=0.47, \alpha_{2}=0.14, c_{1}=0.01, c_{2}=1.5 \times 10^{21}, T=10$, $\theta=2.4680, \delta=0.1$. Then, by solving the linear matrix inequality (41), the corresponding state feedback controller gains are obtained as follows

$$
K_{1}=\left[\begin{array}{lll}
4.4995 & -5.2703 & -2.7031 \\
3.4235 & -9.4527 & -4.9857
\end{array}\right], \quad K_{2}=\left[\begin{array}{ccc}
-14.7294 & 1.8166 & -0.9038 \\
-2.3569 & -0.5674 & -3.3576
\end{array}\right] .
$$




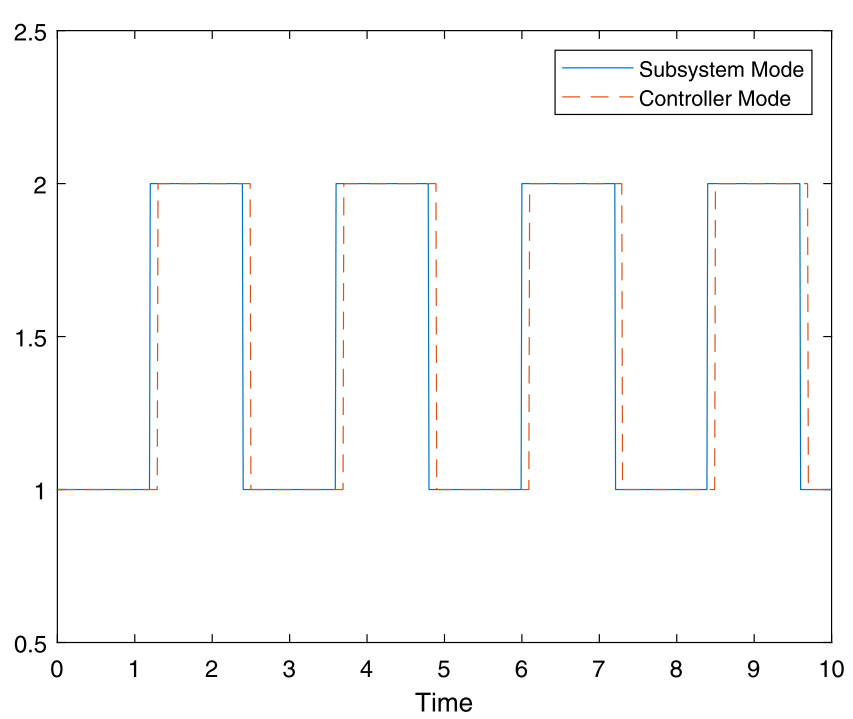

Figure 1 The switching signal

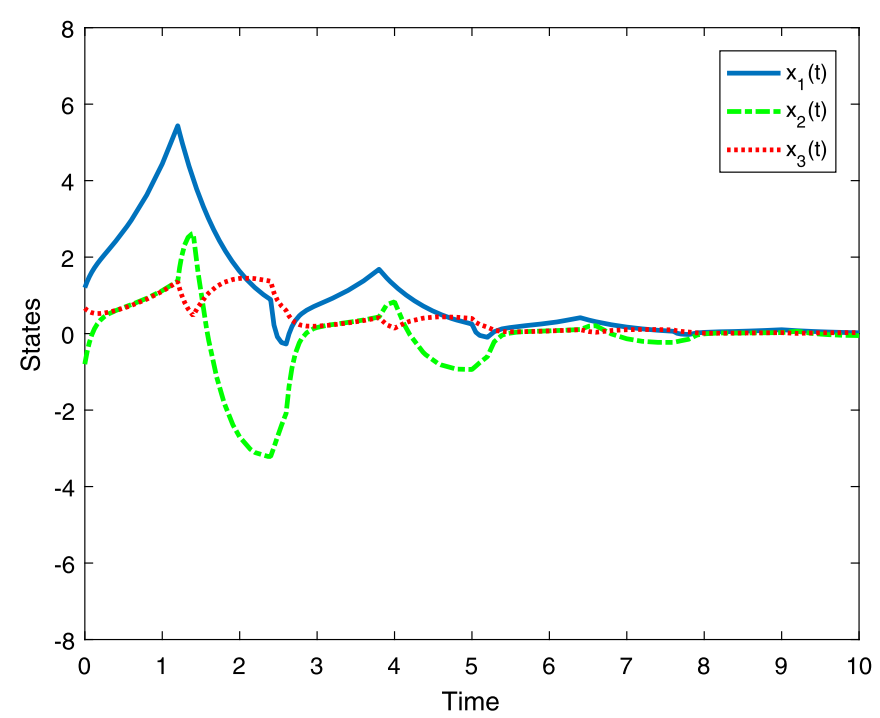

Figure 2 State response of the closed-loop system with $\tau_{a}=1.2, \bar{\tau}_{t}=0.1$

It can be calculated that $\tau_{a}=1.2$. The switching signals of the subsystems and the controllers are plotted in Fig. 1, respectively. Choose the initial state response as $x_{0}=$ $[1.2,-0.8,0.6]$, then the state response of switched singular system (1) under the action of asynchronous controller (3) is depicted in Fig. 2. From the curve in this figure, it can be seen that the three state variables of the system tend to be stable in a finite-time interval under the action of the switching signal designed by Theorem 3.2.

Example 2 Consider a set of 2-dimensional switched singular systems selected from the numerical simulation in reference [25]. The corresponding matrix coefficients are shown 


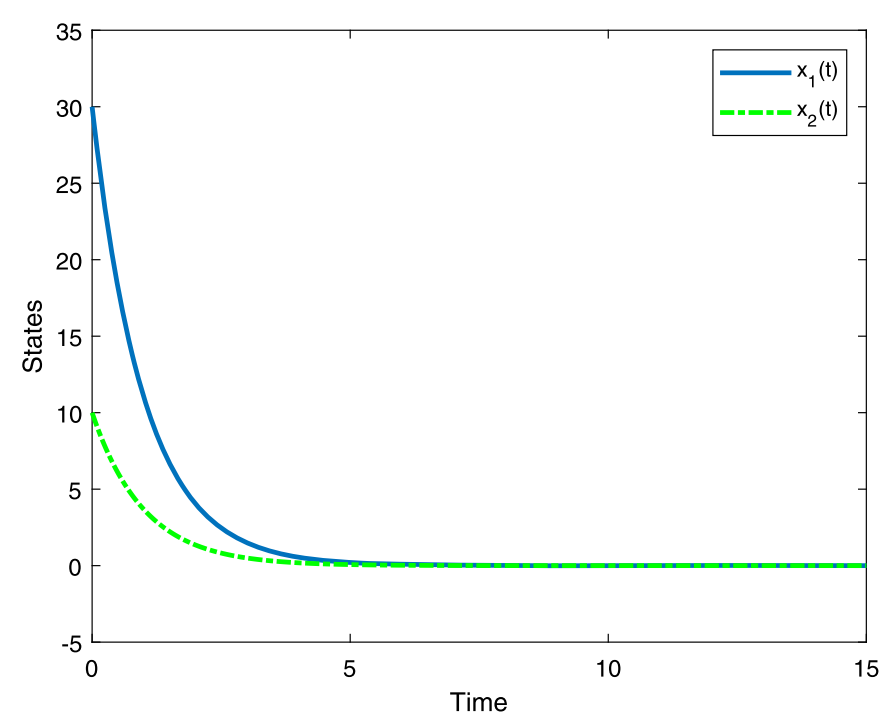

Figure 3 State response of the closed-loop system with $\tau_{a}=0.3$

Table 1 Comparisons of $\tau_{a 1}$ between [25] and Theorem 3.2

\begin{tabular}{lllllll}
\hline$\alpha_{1}$ & 0.17 & 0.26 & 0.47 & 0.52 & 0.58 & 0.68 \\
\hline$\tau_{a 1}$ in [25] & 0.3224 & 0.3357 & 0.3744 & 0.3852 & 0.3991 & 0.4248 \\
$\tau_{a 1}$ in this paper & 0.2656 & 0.2782 & 0.2953 & 0.3084 & 0.3365 & 0.3671 \\
\hline
\end{tabular}

below:

$$
E_{1}\left[\begin{array}{ll}
1 & 1 \\
0 & 0
\end{array}\right], \quad A_{1}=\left[\begin{array}{cc}
-2 & 1 \\
1 & -2
\end{array}\right], \quad E_{2}=\left[\begin{array}{ll}
0 & 1 \\
0 & 0
\end{array}\right], \quad A_{2}=\left[\begin{array}{ll}
0 & 1 \\
1 & 1
\end{array}\right] .
$$

It is easy to verify that subsystem 1 is a stable system and subsystem 2 is an unstable system. The authors of [25] investigated the finite-time stabilization of switched singular linear systems via the Lyapunov approach. In this paper, we study the finite-time stability of linear switched singular systems based on the form of the initial solution of the equation. We choose

$$
M_{1}=M_{2}=I_{2}, \quad N_{1}=\left[\begin{array}{cc}
1 & -1 \\
0 & 1
\end{array}\right], \quad N_{2}=\left[\begin{array}{cc}
1 & -1 \\
1 & 0
\end{array}\right] .
$$

Let $\alpha_{1}=1.5, \alpha_{2}=5, c_{1}=1, c_{2}=30$. The average residence time was calculated to be 0.3 , i.e., less than the time given in [25] of 0.38 . The corresponding state responses of the 2dimensional linear switched singular system are illustrated in Fig. 3. See Table 1 for more comparison of calculation results. It can be seen that tighter dwell bounds are obtained as long as we choose appropriate parameters.

\section{Conclusion}

Finite-time stabilization problems for a class of switched nonlinear singular systems have been discussed in this paper. A controller describing asynchronism has been presented 
and considered in the analysis. By decomposing the system, the regular and impulsefree nature of the switched system is proved to be valid. Without the help of the Lyapunov method, combining the average dwell-time method with differential equation theory, some necessary conditions for finite-time stabilization of systems are given in the form of linear matrix inequalities. In addition, the conditions for solving the parameters of the controller have been obtained. Finally, two numerical examples have been given to verify the effectiveness and correctness of the method presented in this paper. The extensions of the derived results to the finite-time stabilization problem of a fractional-order switched system will be our future investigation.

\section{Acknowledgements}

We would like to express our sincere gratitude to the anonymous referee for his/her helpful comments that helped to improve the quality of the manuscript.

\section{Funding}

Not applicable.

\section{Availability of data and materials}

The authors declare that all data and material in the paper are available and verifitable.

\section{Declarations}

Competing interests

The authors declare that they have no competing interests.

\section{Authors' contributions}

The authors equally conceived of the study, participated in its design and coordination, drafted the manuscript, and read and approved the final manuscript.

\section{Publisher's Note}

Springer Nature remains neutral with regard to jurisdictional claims in published maps and institutional affiliations.

Received: 17 May 2021 Accepted: 24 November 2021 Published online: 07 December 2021

\section{References}

1. Zhang, H.B., Wang, B., Xie, D.H., Xu, S.Y., Yang, C.Y.: Stability, $L_{2}$-gain and asynchronous $H_{\infty}$ control for continuous-time switched systems. Int. J. Robust Nonlinear Control 25, 575-587 (2015)

2. Sun, Z.D.: Robust switching of discrete-time switched linear systems. Automatica 48(1), 239-242 (2012)

3. Li, S., Xiang, Z.R.: Stabilisation of a class of positive switched nonlinear systems under asynchronous switching. Int. J. Syst. Sci. 48(7), 1537-1547 (2017)

4. Xing, M.P., Xia, J.W., Wang, J., Meng, B., Shen, H.: Asynchronous $H_{\infty}$ filtering for nonlinear persistent dwell-time switched singular systems with measurement quantization. Appl. Math. Comput. 362, 1-14 (2019)

5. Ren, H.L., Zong, G.D., Karimi, H.R.: Asynchronous finite-time filtering of networked switched systems and its application: an event-driven method. IEEE Trans. Circuits Syst. I, Regul. Pap. 66(1), 391-402 (2019)

6. Jiao, T.C., Park, J.H., Zong, G.D., Liu, J., Chen, Y.: Stochastic stability analysis of switched genetic regulatory networks without stable subsystems. Appl. Math. Comput. 359, 261-277 (2019)

7. Xue, H.B., Xu, X.H., Zhang, J.Y., Yang, X.P.: Robust stability of impulsive switched neural networks with multiple time delays. Appl. Math. Comput. 359, 456-475 (2019)

8. Zong, G.D., Yang, D.: H-infinity synchronization of switched complex networks: a switching impulsive control method. Commun. Nonlinear Sci. Numer. Simul. 77, 338-348 (2019)

9. Tuan, L.A., Phat, V.N.: Existence of solutions and finite-time stability for nonlinear singular discrete-time neural networks. Bull. Malays. Math. Sci. Soc. 2, 1-20 (2018)

10. Yang, R.M., Sun, L.Y., Zhang, G.Y., Zhang, Q.: Finite-time stability and stabilization of nonlinear singular time-delay systems via Hamiltonian method. J. Franklin Inst. Eng. Appl. Math. 356(12), 5961-5992 (2019)

11. Li, M.Q., Sun, L.Y.: Finite-time stabilisation for a class of nonlinear descriptor systems. IET Control Theory Appl. 12(17), 2399-2406 (2019)

12. Song, X.N., Wang, Z., Shen, H., Li, F., Chen, B., Lue, J.W.: A unified method to energy-to-peak filter design for networked Markov switched singular systems over a finite-time interval. J. Franklin Inst. 354(17), 7899-7916 (2019)

13. Xiao, J.Y., Xu, F.: State bounding estimation for a linear continuous-time singular system with time-varying delay. Adv. Differ. Equ. 120, 1-17 (2019)

14. Zheng, M.J., Yang, S.H., Li, L.N.: Sliding mode control for fuzzy Markovian jump singular system with time-varying delay. Int. J. Control. Autom. Syst. 17(7), 1677-1686 (2019)

15. Zhao, W.Y., Ma, Y.C., Chen, A.H., Fu, L., Zhang, Y.T.: Robust sliding mode control for Markovian jump singular systems with randomly changing structure. Appl. Math. Comput. 349, 81-96 (2019) 
16. Xiao, X.Q., Park, J.H., Zhou, L., Lu, G.P.: New results on stability analysis of Markovian switching singular systems. IEEE Trans. Autom. Control 64(5), 2084-2091 (2019)

17. Liu, G.B., Park, J.H., Xu, S.Y., Zhuang, G.M.: Robust non-fragile $H_{\infty}$ fault detection filter design for delayed singular Markovian jump systems with linear fractional parametric uncertainties. Nonlinear Anal. Hybrid Syst. 32, 65-78 (2019)

18. Long, S.H., Zhong, S.M., Guan, H.B., Zhang, D.: Exponential stability analysis for a class of neutral singular Markovian jump systems with time-varying delays. J. Franklin Inst. 356, 6015-6040 (2019)

19. Wu, Z.G., Shi, P., Shu, Z., Su, H.Y., Lu, R.Q.: Passivity-based asynchronous control for Markov jump systems. IEEE Trans. Autom. Control 62(4), 2020-2025 (2017)

20. Wu, Y.Y., Cao, G.D., Li, Q.B., Alsaedi, A., Alsaadi, F.E.: Finite-time synchronization of uncertain coupled switched neural networks under asynchronous switching. Neural Netw. 85, 128-139 (2017)

21. Li, F.B., Du, C.L., Yang, C.H., Gui, W.H.: Passivity-based asynchronous sliding mode control for delayed singular Markovian jump systems. IEEE Trans. Autom. Control 63(8), 2715-2721 (2018)

22. Ren, H.L., Zong, G.D., Li, T.S.: Event-triggered finite-time control for networked switched linear systems with asynchronous switching. IEEE Trans. Syst. Man Cybern. Syst. 48(11), 1874-1884 (2018)

23. Zhou, L., Ho, D.W.C., Zhai, G.S.: Stability analysis of switched linear singular systems. Automatica 49, 1481-1487 (2013)

24. Wang, Y.J., Zou, Y.C., Liu, Y.H., Shi, X.M., Zuo, Z.Q.: Average dwell time approach to finite-time stabilization of switched singular linear systems. J. Franklin Inst. 352(7), 2920-2933 (2015)

25. Wei, J.M., Zhang, X.X., Zhi, H.M., Mu, X.W., Zhu, X.L.: New finite-time stability conditions of linear switched singular systems with finite-time unstable subsystems. Int. J. Gen. Syst. 48(7), 792-810 (2019)

26. Zong, G.D., Wang, R.H., Zheng, W.X., Hou, L.L.: Finite-time stabilization for a class of switched time-delay systems under asynchronous switching. Appl. Math. Comput. 219(11), 5757-5771 (2013)

27. Cheng, J., Zhu, H., Zhong, S.M., Zheng, F.X., Zeng, Y.: Finite-time filtering for switched linear systems with a mode-dependent average dwell time. Nonlinear Anal. Hybrid Syst. 15, 145-156 (2015)

28. Xiao, J.Y., Wen, S.P., Yang, X.J., Zhong, S.M.: New approach to global Mittag-Leffler synchronization problem of fractional-order quaternion-valued BAM neural networks based on a new inequality. Neural Netw. 122, 320-337 (2020)

29. Xiao, J.Y., Cao, J.D., Cheng, J., Zhong, S.M., Wen, S.P.: Novel methods to finite-time Mittag-Leffler synchronization problem of fractional-order quaternion-valued neural networks. Inf. Sci. 526, 221-244 (2020)

30. Xiao, J.Y., Cheng, J., Shi, K.B., Zhang, R.M.: A general approach to fixed-time synchronization problem for fractional-order multi-dimension-valued fuzzy neural networks based on memristor. IEEE Trans. Fuzzy Syst. https://doi.org/10.1109/tfuzz.2021.3051308(2021)

31. Xiao, J.Y., Cao, J.D., Cheng, J., Wen, S.P., Zhang, R.M., Zhong, S.M.: Novel inequalities to global Mittag-Leffler synchronization and stability analysis of fractional-order quaternion-valued neural networks. IEEE Trans. Neural Netw. Learn. Syst. 32(8), 3700-3709 (2020)

32. Li, X.L., Lin, X.Z., Li, S.H., Zou, Y.: Finite-time stability of switched nonlinear systems with finite-time unstable subsystems. J. Franklin Inst. 352(3), 1192-1214 (2015)

33. Zhang, L.X., Gao, H.J.: Asynchronously switched control of switched linear systems with average dwell time. Automatica 46(5), 953-958 (2010)

34. Wang, Y.E., Sun, X.M., Wu, B.W.: Lyapunov-Krasovskii functionals for switched nonlinear input delay systems under asynchronous switching. Automatica 61, 126-133 (2015)

35. Nguyen, T.: Thanh etc. Finite-time stability of singular nonlinear switched time-delay systems: a singular value decomposition approach. J. Franklin Inst. Eng. Appl. Math. 354(8), 3502-3518 (2017)

36. Hammi, M., Hammami, M.A.: Gronwall-Bellman type integral inequalities and applications to global uniform asymptotic stability. CUBO 17(3), 53-70 (2015)

37. Duan, G.R.: Analysis and Design of Descriptor Linear Systems. Springer, New York (2010)

38. Wang, W., Ma, S.P., Zhang, C.H.: Stability and static output feedback stabilization for a class of nonlinear discrete-time singular switched systems. Int. J. Control. Autom. Syst. 11(6), 1138-1148 (2013)

39. Amato, F., Ariola, M., Dorato, P.: Finite-time control of linear systems subject to paramatric uncertainties and disturbances. Automatica 37(9), 1459-1463 (2001)

\section{Submit your manuscript to a SpringerOpen ${ }^{\circ}$ journal and benefit from:}

- Convenient online submission

- Rigorous peer review

- Open access: articles freely available online

- High visibility within the field

- Retaining the copyright to your article

Submit your next manuscript at $\boldsymbol{s p r i n g e r o p e n . c o m ~}$ 